\title{
A theoretical study of the aromatic character of polyphosphaphospholes. Is the pyramidality the only factor to take into consideration?
}

D. Josa $^{[a]}$, A. Peña-Gallego ${ }^{*}$,[a], J. Rodríguez-Otero ${ }^{[a]}$, and E. M. Cabaleiro$\operatorname{Lago}^{[b]}$

[a] D. Josa, Prof. A. Peña-Gallego, Prof. J. Rodríguez-Otero

Departamento de Química Física, Facultade de Química, Universidade de Santiago de Compostela, Avda. das Ciencias s/n. 15782, Santiago de Compostela, Spain.

fax: $+34-981-595012$

angeles.pena@usc.es

[b] Prof. E. M. Cabaleiro-Lago

Departamento de Química Física, Facultade de Ciencias, Universidade de Santiago de Compostela, Campus de Lugo, Avda. Alfonso X El Sabio s/n, 27002 Lugo, Spain.

\footnotetext{
Abstract: A comprehensive MP2/6-311+G(d,p) and B3LYP/6-311+G(d,p) study of the aromatic character of phospholes, $\mathrm{P}_{n}(\mathrm{CH})_{4-n} \mathrm{PH}$ with $n=0-4$ was conducted. For this purpose, the structures for these compounds were optimized at both theoretical levels and different magnetic properties (magnetic susceptibility, $\chi$, magnetic susceptibility anisotropy, $\chi_{\text {anis, }}$ and the nucleus-independent chemical shifts, NICS) were evaluated. For comparison, these magnetic properties were also calculated in the optimized structures with planarity constraints. We have also applied the ACID (anisotropy of the current-induced density) method in this analysis. The main conclusions are the aromatic character of these compounds, the relationship between aromaticity and planarity and the importance of other factors in this aromaticity.
} 


\section{Introduction}

The backbone of classical heterocyclic chemistry is fundamentally formed by fivemembered heterocycles such us pyrroles, furans, and thiophenes together with sixmembered pyridines. Their chemistry has been continuously developed reaching huge proportions. These molecules have an essential role in biological chemistry and a recent interest for applied chemistry (as example, doped films of polypyrrole have electroconducting properties). However, the chemistry of phospholes has been underdeveloped when compared to its nitrogen, oxygen, and sulphur counterparts. In fact, the first phosphole has been discovered as late as $1959 .{ }^{1,2}$

The aromaticity of the phosphole and its derivatives has been one of their most analyzed properties. $^{3}$ The potential aromaticity of phosphole was discussed after its first practically applicable synthesis ${ }^{4}$ was reported in the 1960s. In different reviews the nonaromatic behaviour of phosphole was indicated. ${ }^{5-7}$ This fact was explained in basis to the pyramidal preference of tricoordinate phosphorus in its compounds. ${ }^{8}$

The planarity of the tricoordinate phosphorus can be influenced by substituents. In this sense, several cases have been studied: $\pi$-acceptor groups either at phosphorus or at the neighbouring carbon, ${ }^{9,10}$ bulky substituents at phosphorus, ${ }^{11-13}$ etc.

Some studies have analyzed the replacement of the $-\mathrm{CH}=$ units in the phosphole by $\mathrm{P}=$, resulting polyphospholes. ${ }^{14,15}$ The main consequence is the decrease of pyramidality and an enhancement of the aromatic character. ${ }^{14}$ The recent interest in compounds with planar or partially planar tricoordinate phosphorus is focused in the relationship between aromaticity and pyramidality. In this article we discuss this fact but also introduce new important effects. 
It is well know that aromatization affects magnetic properties such as magnetic susceptibility and its anisotropy, leading to specially negative values for such properties. ${ }^{16-18}$ These magnitudes are global properties, which can be affected by parts of the molecule not directly implicated in the aromaticity. To avoid this problem, the Nucleus Independent Chemical Shift (NICS) proposed by Schleyer et al. is very useful. The NICS is defined as the negative of the magnetic shielding ${ }^{19}$ and it can be evaluated at any point of the molecule, exhibiting very negatives values in the center of aromatic rings.

The ACID (anisotropy of the current-induced density) is a new method based on magnetic properties and developed by Herges and Geuenich. ${ }^{20}$ This method allows the visualization of the ring current formed when a magnetic field is applied and permits us to study the electronic delocalization in molecules. ${ }^{21,22}$

\section{Computational details:}

The geometry of each structure was optimized with the $6-311+G(d, p)$ basis set and the density functional theory (specifically, the Becke3LYP functional) ${ }^{23,24}$ or MøllerPlesset perturbation level with the inclusion of energy corrections through second-order (MP2). All structures were minima as frequency calculations at same level have shown. In order to evaluated the relationship of planarity with aromaticity, geometries of $1-4 b$ structures with planarity constraints were optimized at the B3LYP and MP2 level with 6-311+G(d,p) basis set. These planar structures were transition states.

The anisotropy of magnetic susceptibility values was calculated at the B3LYP/6$311+\mathrm{G}(\mathrm{d}, \mathrm{p})$ level using the IGAIM (Individual Gauges for Atoms in Molecules) method $^{25,26}$ on the B3LYP and MP2 optimized structures. In the NICS calculations, 
B3LYP/6-311+G(d,p) level with GIAO (Gauge-independent Atomic Orbital) method ${ }^{27}$ were employed.

Finally, CSGT (Continuous Set of Gauge Transformations) method ${ }^{25,26,28}$ at B3LYP/6$31+\mathrm{G}(\mathrm{d})$ level of theory was employed in ACID calculations, carried out with the program supplied by Herges. ${ }^{20}$

Quantum chemical calculations were carried out with the Gaussian $98^{29}$ and Gaussian $03^{30}$ program packages.

\section{Results and discussion:}

Figure 1 shows the polyphosphaphospholes studied in the present work. These structures, except for the pentaphosphole (5), are no planar. Table 1, collects the pyramidality data of these structures. The values for different magnetic properties: anisotropy of the susceptibility (we have chosen this property instead of magnetic susceptibility due to the inherent problems of this property ${ }^{31}$ ) and NICS are shown in the same Table 1. The NICS criterion is based on the negative of the magnetic shielding computed at the centre of the ring. Negative values imply aromaticity (diatropic ring current) and positive values imply antiaromaticity (paratropic ring current). The NICS values have been obtained at ring critical point as defined by Bader. ${ }^{32}$ NICS values at points in the ring plane (NICS(0)) contain important spurious contributions from the inplane tensor components that are not related to aromaticity as Schleyer has indicated. ${ }^{33}$ For this reason, NICS(1) and NICS(-1) values (1 $\AA$ above/below the plane of the ring) are showed in Table 1 . These values reflect $\pi$ effects and they are a better indicator of the ring current than values in ring plane, because at $1 \AA$ the effects of the local $\sigma$ bonding contributions are diminished. 
In this sense and in order to obtain a better knowledge about the aromaticity of phosphole and derivatives, we have also computed the NICS values in points above and below the plane of the molecule (z-axis). As we can observe in Figure 2, the variation of NICS along the $z$-axis is very similar for all compounds. This representation are in agreement with a $\pi^{2}$ aromaticity. ${ }^{34,35}$ In the usual $\pi$ aromaticity the maximum diamagnetic shielding takes place at a certain distance above and below the molecular plane, as in this case. This effect can be described by means of two ring currents circulating at a certain distance from the molecular plane. The figures also indicate the higher aromatization in the opposite side to the hydrogen atom joined to the phosphorus atom. These figures are a clear evidence of the aromatic character of phosphole and derivatives contrary to some studies that suggest a nonaromatic or only slight aromatic character.

Different studies have looked for a direct connection between planarity and aromaticity in these compounds. In this sense, the search of more aromatic derivatives of the phosphole was reduced many times by the search of substituents that produced more planar structures. In this work we are interested in investigating this connection between planarity and aromaticity and if other factors should be considered. For this purpose NICS values all compounds are presented in Table 1 together with pyramidality data. The pyramidality at the tricoordinate phosphorus atom is measured by the sum of the bond angles, $\Sigma \alpha$. At first sight a good correlation between planarity and aromaticity is observed. Even so, some details attract attention: the structures for $2 \mathrm{a}$ and $2 \mathrm{~b}$ at B3LYP/6-31++G(d,p) level have very similar planarity values but its NICS(0) values differ in nearly two ppm, or, in the case of structures with three phosphorus atoms, 3a and $3 c$ have similar $\Sigma \alpha$ but very different $\operatorname{NICS}(0)$ values. In order to visualize the correlation between pyramidality and aromaticity, the anisotropy and NICS(1) and 
NICS(-1) values for all polyphosphaphospholes are plotted against the bond angle sums (Figure 3). A relation between pyramidality and aromaticity is present but some facts seem indicate that a deeper analysis is necessary. If the four compounds with three phosphorus atoms are selected, we can observe that NICS(1) and NICS(-1) values for $3 \mathrm{a}$ are more negative than expected values if a perfect correlation between pyramidality and aromaticity exists at both DFT and MP2 method. This result seems to indicate that the extension of conjugation is greater when the phosphorus atoms are connected and the $\mathrm{P}-\mathrm{H}$ group is in the end of this connection.

\begin{tabular}{|c|c|c|c|c|c|c|c|c|c|c|}
\hline \multirow{2}{*}{} & \multicolumn{2}{|c|}{$\Sigma \alpha\left({ }^{\circ}\right)$} & \multicolumn{2}{c|}{$\chi_{\text {anis }}$ /cgs-ppm } & \multicolumn{2}{c|}{ NICS (0)/ppm } & \multicolumn{2}{c|}{ NICS (1)/ppm } & \multicolumn{2}{c|}{ NICS (-1)/ppm } \\
\cline { 2 - 11 } & DFT & MP2 & DFT & MP2 & DFT & MP2 & DFT & MP2 & DFT & MP2 \\
\hline 1 & 292.4 & 294.1 & -42.5 & -44.0 & -5.9 & -6.2 & -5.6 & -5.9 & -6.1 & -6.5 \\
\hline $2 \mathrm{a}$ & 300.7 & 308.8 & -53.2 & -54.6 & -7.8 & -8.7 & -7.2 & -7.8 & -7.4 & -8.4 \\
\hline 2b & 300.4 & 302.6 & -50.8 & -52.5 & -5.6 & -6.0 & -6.7 & -7.1 & -7.2 & -7.6 \\
\hline 3a & 309.1 & 318.0 & -65.9 & -67.9 & -8.3 & -9.6 & -8.2 & -9.0 & -8.9 & -9.9 \\
\hline 3b & 312.6 & 321.4 & -63.5 & -66.0 & -7.8 & -9.2 & -8.3 & -9.2 & -8.8 & -9.9 \\
\hline 3c & 316.8 & 328.0 & -67.3 & -68.7 & -9.6 & -11.1 & -9.0 & -9.9 & -9.1 & -10.4 \\
\hline $3 \mathrm{~d}$ & 310.9 & 317.2 & -64.4 & -67.6 & -6.7 & -7.8 & -7.9 & -8.7 & -8.6 & -9.4 \\
\hline 4a & 324.6 & 337.8 & -83.1 & -87.4 & -10.2 & -12.7 & -10.0 & -11.3 & -11.0 & -12.5 \\
\hline 4b & 329.0 & 339.7 & -83.7 & -85.8 & -11.3 & -13.0 & -10.7 & -11.6 & -11.2 & -12.4 \\
\hline 5 & 360.0 & 359.9 & -113.0 & -111.9 & -17.7 & -17.6 & -15.1 & -15.0 & -15.1 & -15.2 \\
\hline
\end{tabular}

Table 1. Values of $\Sigma \alpha$ and magnetic properties for the optimized structures at B3LYP and MP2 level.

Similar behaviour is observed if compounds with two atoms of phosphorus are analyzed. So, the compound $2 \mathrm{a}$ with the phosphorus atom linked to P-H group is more aromatic than compound $2 \mathrm{~b}$, even though at B3LYP level the planarity of their structures is very similar.

We should remember that the evaluation of the absolute aromaticity of a compound remains a controversial issue. ${ }^{36}$ The main reason to this affirmation with regard to the 
magnetic properties as indication of aromatic character is the lack of a reference. For this reason, we have thought in the use of some system as reference value. Many of previous works have analyzed the relationship between pyramidality and aromaticity, so we have chosen the planar structures corresponding to each system as reference structure. These planar structures are transition states. Table 2 shows the differences $(\triangle N I C S=N I C S$ (pyramidal compound) - NICS (planar compound) $)$ of the magnetic properties between the planar and pyramidal structures. As for the absolute values of magnetic properties, a direct relationship between pyramidality and aromaticity is complicated. So, one of the structures with a smaller pyramidality $(\Sigma \alpha=337.8)$, the compound $4 \mathrm{a}$, presents values even bigger than $3 \mathrm{c}$, a structure with $\Sigma \alpha=328.0$. But, when the distance between phosphorus atoms is analyzed, the patron is the same indicated for the absolute values of NICS and even the differences are more significant (for example, the difference between $\triangle N I C S$ for structure $3 \mathrm{c}$ and $3 \mathrm{~d}$ is more than 3 ppm). The increase of this effect may indicate that the interaction between phosphorus atoms is larger in pyramidal structures than in planar structures.

\begin{tabular}{|c|c|c|c|c|}
\hline \multirow{2}{*}{} & \multicolumn{2}{|c|}{$\Delta N I C S(1) / \mathrm{ppm}$} & \multicolumn{2}{c|}{$\Delta N I C S(-1) / \mathrm{ppm}$} \\
\cline { 2 - 5 } & DFT & MP2 & DFT & MP2 \\
\hline 1 & 5.0 & 4.8 & 4.5 & 4.2 \\
\hline 2a & 4.6 & 4.0 & 4.4 & 3.4 \\
\hline 2b & 4.4 & 4.1 & 3.9 & 3.5 \\
\hline 3a & 4.6 & 3.8 & 3.9 & 2.9 \\
\hline 3b & 3.9 & 3.1 & 3.4 & 2.4 \\
\hline 3c & 3.5 & 2.6 & 3.4 & 2.2 \\
\hline 3d & 4.3 & 3.8 & 3.6 & 3.1 \\
\hline 4a & 3.8 & 2.5 & 2.8 & 1.3 \\
\hline 4b & 2.9 & 1.9 & 2.4 & 1.2 \\
\hline
\end{tabular}

Table 2. Difference between NICS for planar and pyramidal structures. 
In order to carry out a deeper study of the aromaticity of the phosphole and these derivatives, the ACID method was employed. This is a relatively recently published method to investigate the delocalization and conjugation effects in molecules. It provides a powerful way to visualize the density of delocalized electrons and quantify conjugation effects. The ACID approach has several advantages: it is a scalar field which is invariant with respect to the relative orientation of the magnetic field and the molecule, it is not a simple function of the overall electron density, it has the same symmetry as the wave function, and it can be plotted as an isosurface. In our group this method has been extensively employed in order to distinguish between pericyclic/pseudopericyclic and coarctate/pseudocoarctate reactivity, differentiation where the aromaticity has a crucial paper.

Figure 4 presents the ACID isosurface of the studied polyphosphaphospholes at an isosurface value of 0.03 au. Current density vectors are plotted onto the ACID isosurface. The first point to emphasize is that Figure 4 shows a strong diatropic ring current for all these compounds, indicating their aromatic character. This affirmation is opposite to other studies that have indicated that the pyramidality of phosphole prevented the aromaticity. In this figure the importance of the position of the $-\mathrm{P}=$ units with regard to the PH group is clearly shown. In this sense, the most obvious case is the comparison between $3 \mathrm{c}$ and $3 \mathrm{~d}$. In the last case the ring current in the $\mathrm{P}=\mathrm{P}$ zone is smaller than in other area of the structure. This point may be more clearly observed if the critical isosurface values, CIV's, are compared. The CIV's indicate the isosurface value at which the topology changes from cyclic to noncyclic. Large CIV's show aromaticity or antiaromaticity and small CIV's show disconnections. So, this values are a good tool to quantify the extent of conjugation. In this case and as an example, the structure $3 \mathrm{~d}$ exhibits the smallest CIV (0.034) indicating a smaller aromaticity than 
other structures with similar $\Sigma \alpha$ ( the CIV for the 3 a structure is 0.041 ). This result is in agreement with result obtained in the analysis of the NICS values and the important role of the position of $\mathrm{P}$ atoms.

\section{Conclusions:}

This work allows to us to indicate the aromatic character of the phosphole and the studied polyphosphaphospholes. This aromaticity is shown by the negative values of the magnetic properties, the representation of the variation of NICS along the $z$-axis, and the ACID figures.

It is also interesting to notice the qualitative agreement between MP2 and DFT results. in order to avoid the problem of lack of reference of the magnetic properties as indicator of the aromaticity, a model was employed: the magnetic properties of the structures are compared with the magnetic properties of these planar structures.

The connection between aromaticity and pyramidality is demonstrated through graphics of magnetic properties versus $\Sigma \alpha$.

But the main conclusion of this work is the importance for the aromaticity of these compounds of other aspects apart from the pyramidality. In this sense, the influence of the position of $-\mathrm{P}=$ units has been clearly indicated. 


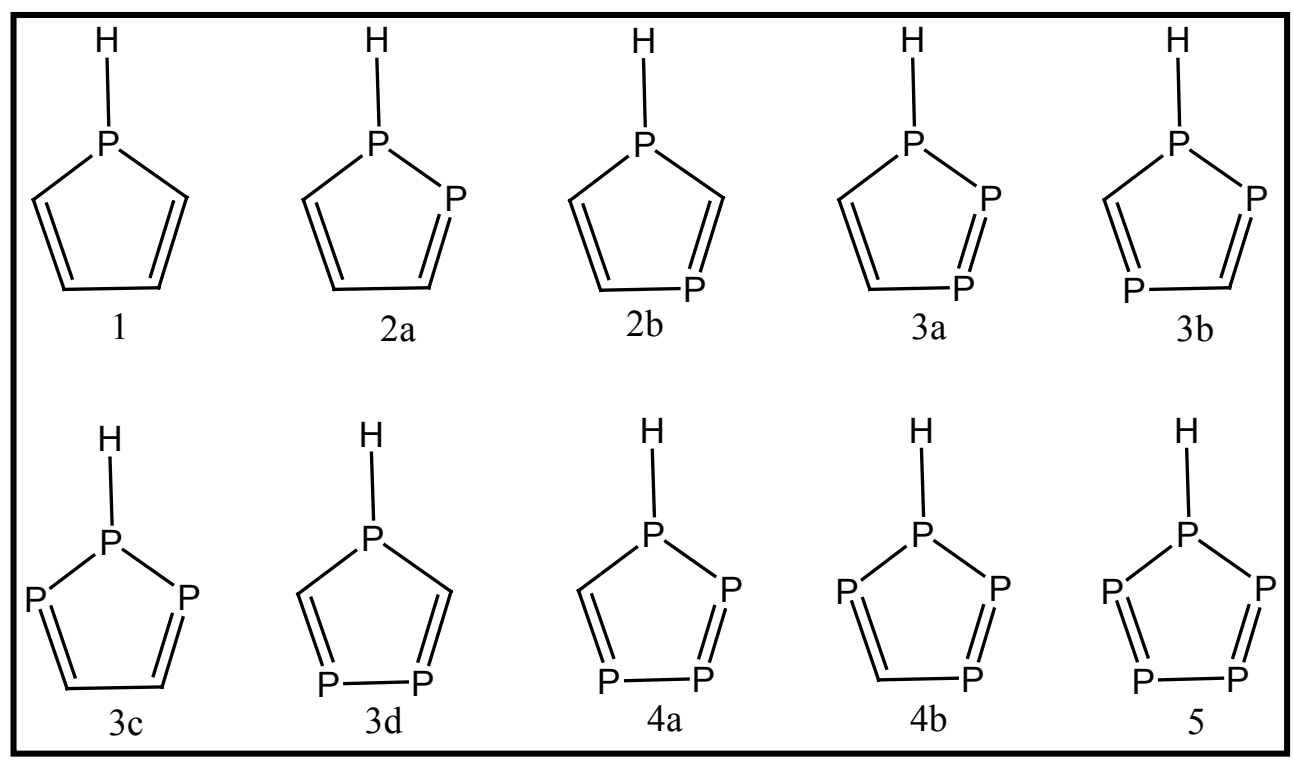

Figure 1. The polyphosphaphospholes object of the study. 


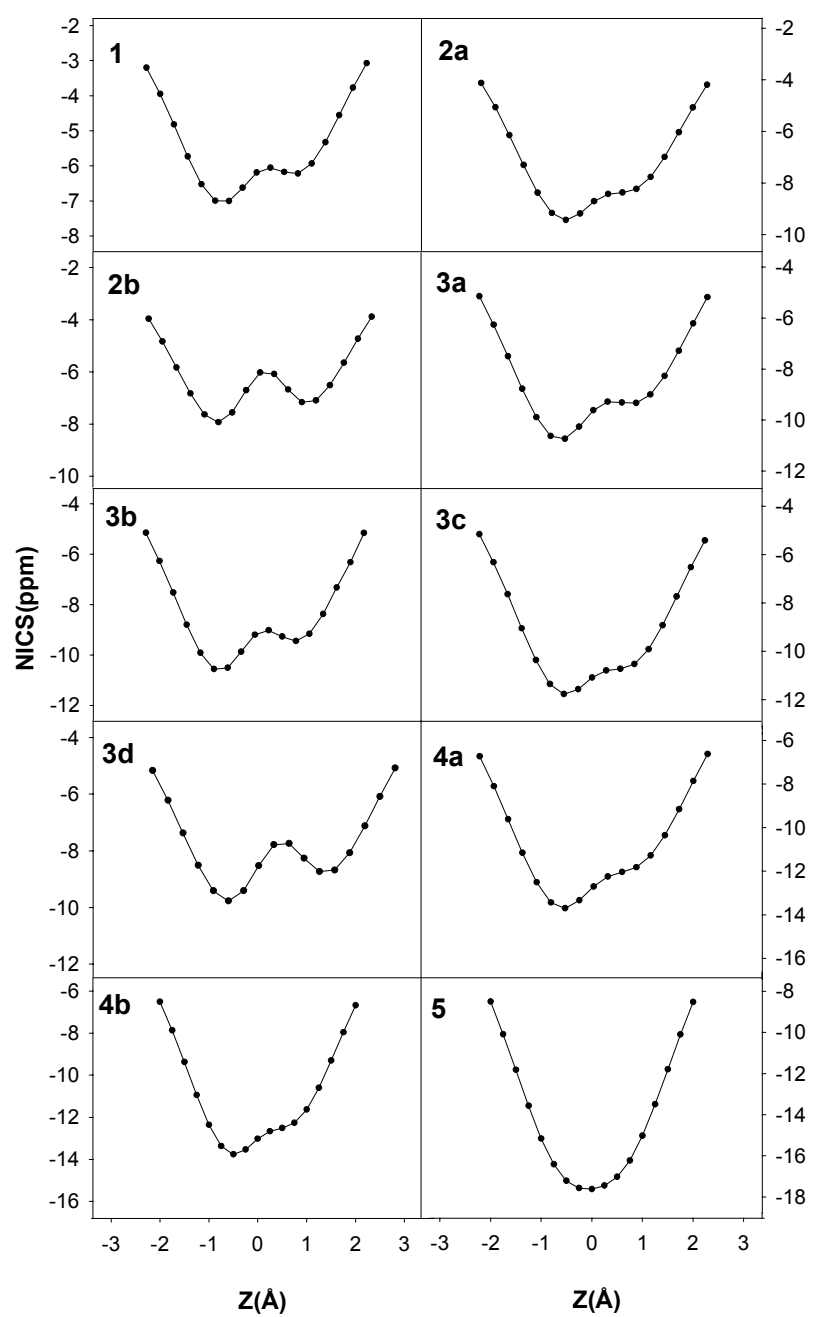

Figure 2. Variation of NICS along z-axis. 


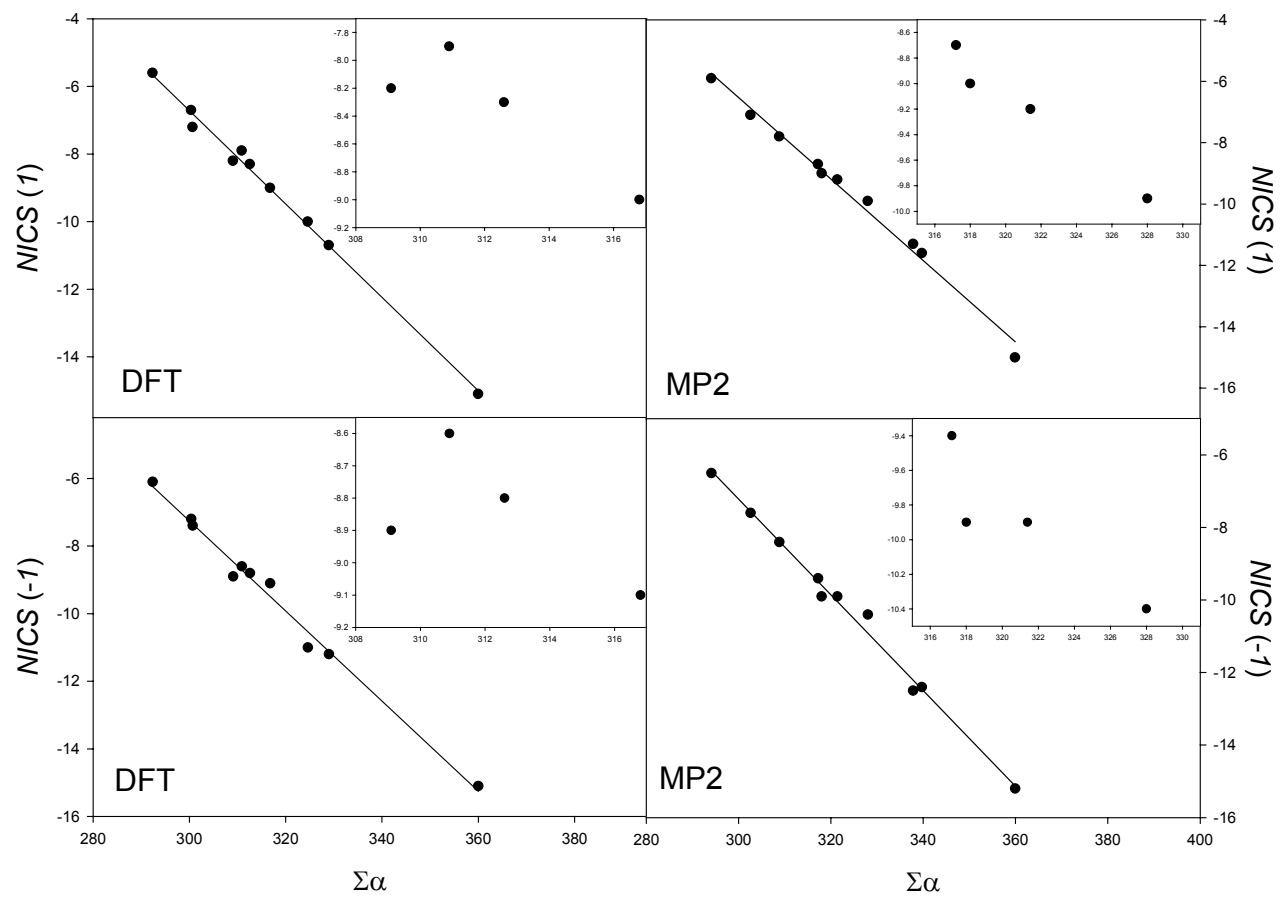

Figure 3. Plots of NICS values versus $\Sigma \alpha$. The area corresponding to structures $3 a, 3 b$, $3 c$ and $3 d$ is magnified. 


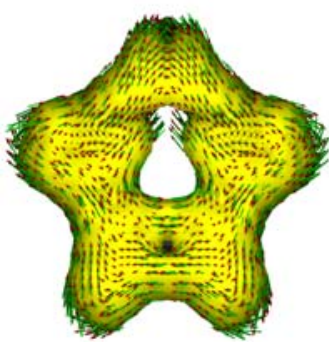

1

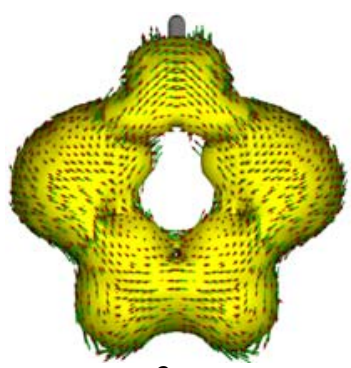

$3 c$

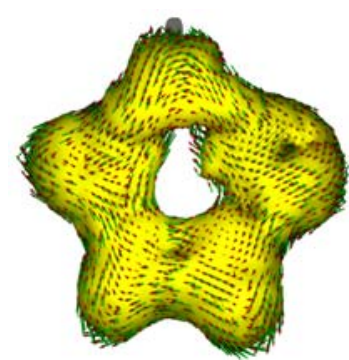

$2 a$

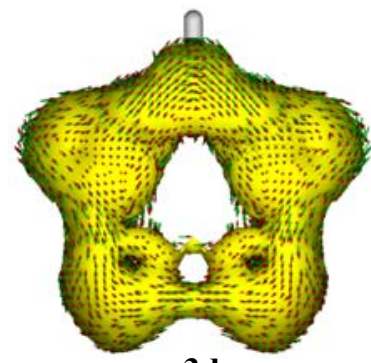

3d

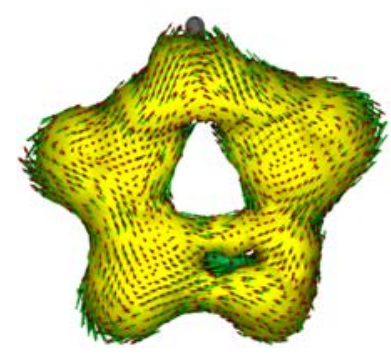

2b

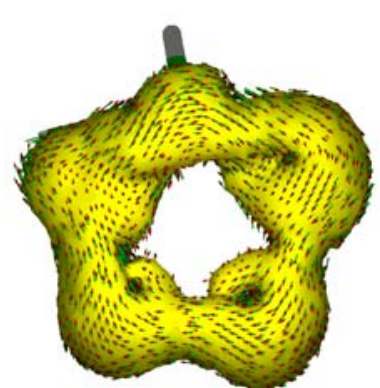

$4 a$

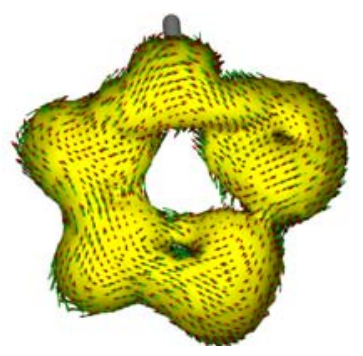

$3 a$

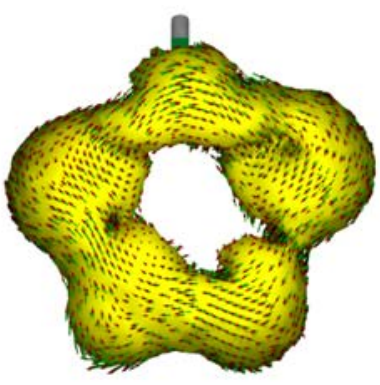

$4 b$

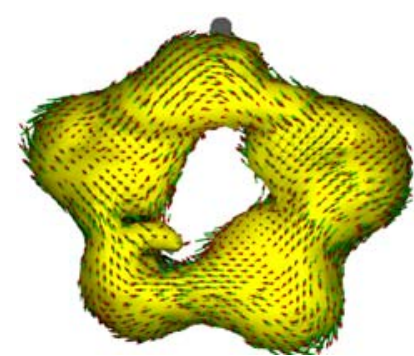

3b

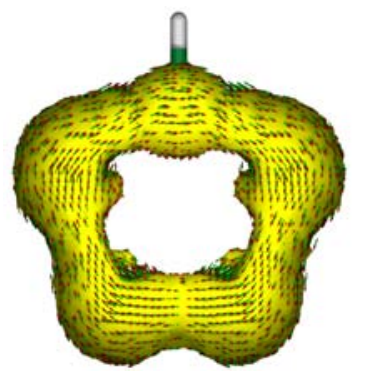

5

Figure 4. ACID figures for the studied structures. 


\section{References:}

1 Braye, E. H.; Hübel, W. Chem. Ind. (London) 1959, 1250.

2 Leavitt, F. C.; Manuel, T. A.; Johnson, F. J. Am. Chem. Soc. 1959, 81, 3163.

3 Nyulászi, L. Chem. Rev. 2001, 101, 1229.

4 a) Quin, L. D.; Bryson, J. G. J. Am. Chem. Soc. 1967, 89, 5984. b) Mathey, F. C. R. Acad. Sci. Ser. C 1969, 269, 1066.

5 Mathey, F. Chem. Rev.1988, 88, 437.

6 Hughes, A. N. Phospholes and related Compounds. In Handbook of Organophosphorus Chemistry. Engel, R., Ed.; Marcel Dekker: New York, 1992.

7 Quin, L. D.; Hughes, A. N. Cyclic Phosphines. In The Chemistry of Organophosphorus Chemistry. Hartley, F., Ed.; John Wiley: Chichester, 1990.

8 Coggon, P.; Engel, J. F.; McPhail, A. T.; Quin, L. D. J. Am. Chem. Soc. 1970, 92, 5779.

9 Nyulászi, L. J. Phys. Chem. 1995, 99, 586.

10 Delaere, D.; Dransfeld, A.; Nguyen, M. T.; Vanquickenborne, L. G. J. Org. Chem. 2000, 65, 2631.

11 Quin, L. D.; Keglevich, Gy.; Ionkin, A. S.; Kalgutkar, R.; Szalontai, G. J. Org. Chem. 1996, $61,7801$.

12 Keglevich, Gy.; Böcskei, Zs.; Keserü, Gy.; Ujszászi, K.; Quin, L. D. J. Am. Chem. Soc. 1997, 119, 5095.

13 Nyulászi, L.; Soós, L. Keglevich, Gy. J. Organomet. Chem. 1998, 566, 29.

14 Dransfeld, A.; Nyulászi, L.; Schleyer, P. v. R. Inorg. Chem. 1998, 37, 4413.

15 Nyulászi, L. J. Phys. Chem. 1996, 100, 6194.

16 Zimmermann, H. E. Acc. Chem. Res. 1971, 4, 271.

17 Herges, R.; Jiao, H.; Schleyer, P. v. R. Angew. Chem., Int. Ed. 1994, 33, 1376.

18 Jiao, H.; Schleyer, P. v. R. J. Org. Chem. Phys. Chem. 1998, 11, 655.

19 Schleyer, P. v. R.; Maerker, C.; Dransfeld, A.; Jiao, H; Hommes, N. J. R. v. E. J. Am. Chem. Soc. 1996, 118, 6317.

20 Herges, R. Geuenich, D. J. Phys. Chem. A 2001, 105, 2314. 
21 Herges, R; Papafilippopoulos, A. Angew. Chem., Int. Ed. 2001, 40, 4671.

22 Kimball, D. B.; Weakley, T. J. R.; Herges, R.; Haley, M. M.; J. Am. Chem. Soc. 2002, 124, 13463.

23 Becke, A. D. J. Chem. Phys. 1993, 98, 5648.

24 Lee, C.; Yang, W.; Parr, R. G. J. Phys. Rev. B 1988, 37, 785.

25 T. A. Keith, R. F. W. Bader, Chem. Phys. Lett. 1993, 210, 223.

26 T. A. Keith, R. F. W. Bader, Chem. Phys. Lett. 1992, 194, 1.

27 K. Wolinski, J. F. Hilton, P. Pulay, J. Am. Chem. Soc. 1990, $112,8251$.

28 J. R. Cheeseman, M. J. Frisch, G. W. Trucks, T. A. Keith, J. Chem. Phys. 1996, 104, 5497.

29 M. J. Frisch, G. W. Trucks, H. B. Schlegel, G. E. Scuseria, M. A. Robb, J. R. Cheeseman, V.

G. Zakrzewski, J. A. Montgomery, R. E. Stratmann, J. C. Burant, S. Dapprich, J. M. Millam, A.

D. Daniels, K. N. Kudin, M. C. Strain, O. Farkas, J. Tomasi, V. Barone, M. Cossi, R. Cammi,

B. Mennucci, C. Pomelli, C. Adamo, S. Clifford, J. Ochterski, G. A. Petersson, P. Y. Ayala, Q.

Cui, K. Morokuma, D. K. Malick, A. D. Rabuck, K. Raghavachari, J. B. Foresman, J.

Cioslowski, J. V. Ortiz, B. B. Stefanov, G. Liu, A. Liashenko, P. Piskorz, I. Komaromi, R.

Gomperts, R. L. Martin, D. J. Fox, , T. Keith, M. A. Al-Laham, C. Y. Peng, A. Nanayakkara, C.

Gonzalez, M. Challacombe, P. M. W. Gill, B. G. Johnson, W. Chen, M. W. Wong, J. L. Andres,

M. Head-Gordon, E. S. Replogle, and J. A. Pople, Gaussian, Inc., Pittsburgh, PA, 1998.

30 M. J. Frisch et al., GAUSSIAN 03 (Revision B. 03), Gaussian, Inc., Pittsburgh, PA, 2003.

31 E. M. Cabaleiro-Lago, J. Rodríguez-Otero, S. M. Varela-Varela, A. Peña-Gallego, J. M. Hermina-Ramón, J. Org. Chem. 2005, 70, 3921.

32 R. F. W. Bader, Atoms in Molecules-A Quantum Theory; Clarendon Press: Oxford, 1990.

33 Schleyer, P. v. R.; Manoharan, M.; Wang, Z.-X.; Kiran, B.; Jiao, H.; Puchta, R.; Hommes, N.

J. R. v. E. Org. Lett. 2001, 3, 2465.

34 de Lera, A. R.; Alvarez, R.; Lecea, B; Torrado, A.; Cossío, F. P. Angew. Chem., Int. Ed. $2001,40,557$. 
35 Alajarin, M.; Sánchez-Andrada, P: Cossio, F. P.; Arrieta, A.; Lecea, B. J. Org. Chem. 2001, 66,8470 .

36 See special issue 5 entirely dedicated to aromaticity: Chem. Rev. 2001, 101. 